\title{
A Structured ElGamal-Type Multisignature Scheme
}

\author{
Mike Burmester ${ }^{1}$, Yvo Desmedt ${ }^{2}$, Hiroshi Doi ${ }^{3}$, Masahiro Mambo ${ }^{4}$, \\ Eiji Okamoto ${ }^{5}$, Mitsuru Tada ${ }^{6}$, and Yuko Yoshifuji ${ }^{6, *}$ \\ 1 Information Security Group, Royal Holloway, University of London \\ m. burmester@rhbnc.ac.uk \\ 2 Department of Computer Science, Florida State University \\ desmedt@cs.fsu.edu \\ 3 Department of Mathematics, Faculty of Science, Okayama University \\ hdoi@math.okayama-u.ac.jp \\ 4 Education Center for Information Processing \& Graduate School of Information \\ Sciences, Tohoku University, mambo@ecip.tohoku.ac.jp \\ 5 Center for Cryptography, Computer and Network Security \\ University of Wisconsin, Milwaukee, okamoto@cs. uwm.edu \\ 6 School of Information Science, Japan Advanced Institute of Science and Technology, \\ $\{m t, y o s i f u j i\} @ j a i s t . a c . j p$
}

\begin{abstract}
We propose a structured multisignature scheme which is based on a modified ElGamal signature scheme and analyze its security. The structure takes into account the order of the signers. With serial structures, different signing orders produce different multisignatures. In contrast, with parallel structures the multisignatures are independent of the signing order. Our structured multisignatures can deal with structures which are composed of serial and parallel signing orders. We give reductions for the security of the proposed scheme, and for the specified order of the signers in the serial and mixed cases.
\end{abstract}

Keywords: Multisignature, Structured multisignature, Group structure, Series-parallel graph, ElGamal signature.

\section{Introduction}

When multiple entities sign a document, the signing order often reflects the role of each signer and signatures with different signing orders are regarded as multisignatures with different meanings. A typical example is a multisignature in a company. Usually, the head of a section should sign a document after the other members of the section have signed it. Other examples involve banks and command structures.

Of course the signing order is of little relevance to authentication. However there are other aspects one should take into account, such as the liability of the

${ }^{*}$ Present affiliation of the last author: NTT Service Integration Laboratories 
signers. This could be related to their ranking and determined by the signing order. Depending on the application, a different signing order may be required. Moreover, each signer may only wish to sign after the previous signers have done so, and the verifier may require that the correct order has been adhered to.

We can consider two cases for the signing order: 1) serial signing, in which the signing order can be detected by a verifier from a signature, 2) parallel signing, in which the signing order cannot be detected by a verifier from a signature. A multisignature scheme is said to be structured if the group of signers is structured. The structure takes into account the signing order of the entities of the signing groups, if this is serial. In this case different signing orders yield different multisignatures. In contrast, the multisignature for parallel signing orders is group independent. Our structured multisignature scheme can deal with group structures which are composed of serial and parallel signing orders.

In this paper we propose a structured multisignature scheme based on a modified ElGamal signature scheme and give reductions for its security, including the security for the specified order of the signers. This paper is organized as follows. After this introduction we discuss related work in Section 2. In Section 3 we represent signer groups by series-parallel graphs and in Section 4 we give definitions and specify our setting. In Section 5 we define our model, and the reductions and functions which we shall use for the security proof. After describing the basic modified ElGamal signature scheme in Section 6, we present a serial multisignature scheme in Section 7, a parallel multisignature scheme in Section 8, and a mixed multisignature scheme in Section 9. In Section 10 we modify our scheme to get a more efficient scheme by exploiting the decomposition tree of series-parallel graphs. Finally, conclusions are given in Section 11.

\section{Related Work}

So far there are several proposals for multisignature schemes, as for example in [Oka88,OO91,HZ92,Shim94,DOMU94,HMP95,DOM98,OO99]. Multisignature schemes have advantages over multiple iterations of a single signature scheme either in the length of the signature or in the computational cost of generating and verifying the signature. Another property one should pay attention to is the signing order. Some multisignature schemes are independent of the order of the signers [OO91,HZ92,Shim94,HMP95], while other schemes are order specified [Oka88,DOMU94,DOM98,0099].

Among the schemes which are sensitive to the signing order, the scheme in [Oka88] is constructed using bijective functions such as the RSA signing function [RSA78]. The scheme in [DOMU94] is also based on the RSA signature scheme, but this scheme has been shown to be insecure. A modified version of this scheme is proposed in [DOM98], however its security analysis is not complete. Another scheme [DOM98] uses an ElGamal-type signature [ElG85,HMP94], but as with the RSA based scheme, its security analysis is not complete.

The Ohta-Okamoto schemes in [OO91] are converted from corresponding identification schemes such as the Schnorr scheme [Schn91] and the Fiat-Shamir 
scheme [FS86]. For single signatures, the security proof for the Schnorr signature scheme and a modified ElGamal signature scheme are given in [PS96] using the Random Oracle model [BR93] (however there are some problems with this model [CGH98]). Ohta-Okamoto prove the security of their schemes using an ID reduction to an identification scheme [OO91]. For single signatures their proof gives a tighter reduction than [PS96]. However the Ohta-Okamoto schemes do not consider mixed structures composed of serial and parallel signing orders. In particular, the security of multisignature schemes for such a composite group structure has not been proven.

A structured multisignature scheme based on an ElGamal-type signature is also proposed in [DOM98] but its security for the signing order is not complete.

\section{Series-Parallel Graph}

We represent the group of signers by a graph. We consider directed graphs which satisfy the following conditions:

(G-1) Each signer corresponding to an edge appears only once in the graph.

(G-2) The graphs are restricted to series-parallel type graphs.

A series-parallel graph is a graph which is generated by series and parallel compositions of series-parallel graphs. The simplest series-parallel graph is a base graph of two vertices and an edge. We refer the reader to [Len90] for more details. The set of all graphs satisfying the above conditions is denoted by $S P G$.

In Figure 1 we illustrate a typical series-parallel graph. A series-parallel graph can be decomposed into a unique tree called the decomposition tree of the graph. In Figure 2 we show the decomposition tree of the graph in Figure 1. The labels of the vertices show the type of composition of the subordinate edges. Vertices labeled $S C$ show series compositions whereas vertices labeled $P C$ show parallel compositions. Series-parallel graphs can be recognized in linear time and the decomposition tree of a series-parallel graph can be found in linear time [Len90].

\section{Notation and Definition}

We will use a discrete logarithm setting. Let $p$ and $q$ be appropriate primes such that $q \mid(p-1)$, and let $g \in \mathrm{Z}_{p}^{*}$ be a $q$-th root of 1 modulo $p . P_{i}$ is a signer with secret key $a_{i} \in \mathrm{Z}_{q}^{*}$, and $k_{i}$ is a random number in $\mathrm{Z}_{q}^{*}$ selected by $P_{i} . M \in\{0,1\}^{*}$ is the message to be signed. $a \in_{R} A$ indicates that the element $a$ is selected from the set $A$ uniformly at random.

Constructible groups. Let $\mathcal{G} \subset S P G$ be a collection of signer groups, $G_{k} \in \mathcal{G}$ a signer group represented by a series-parallel graph, $P_{i}$ a signer in $G_{k}$, $a_{i} \in \mathrm{Z}_{q}^{*}$ the secret key of $P_{i}$, and $y_{i}^{(k)} \in \mathrm{Z}_{p}^{*} \backslash\{1\}$ the partial public key of $P_{i}$ corresponding to $a_{i}$. We say that $\mathcal{G}$ is an available group of a signature 


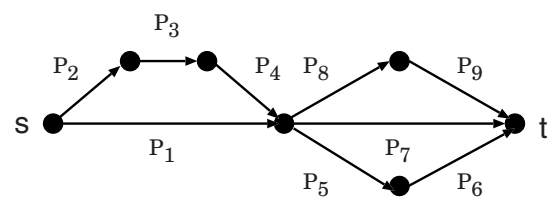

Fig. 1. A series-parallel graph $G$

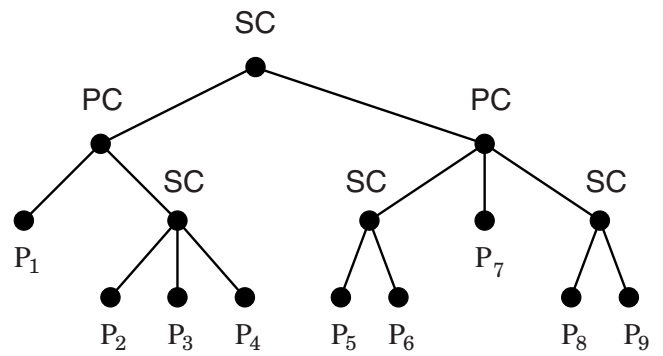

Fig. 2. The decomposition tree of $G$

scheme if there is a set of secret keys $\left\{a_{i} \mid P_{i} \in G_{k}, G_{k} \in \mathcal{G}\right\}$ of this scheme such that,

$$
y_{i}^{(k)} \neq y_{j}^{(l)} \text { for } \forall G_{k}, G_{l} \in \mathcal{G}, \forall\left(P_{i}, P_{j}\right) \in\left(G_{k}, G_{l}\right), i \neq j .
$$

We allow $k=l$ in (1). The set of keys $\left\{a_{i} \mid P_{i} \in G_{k}, G_{k} \in \mathcal{G}\right\}$ of an available group is called an appropriate secret-key set for $\mathcal{G}$ or simply, appropriate for $\mathcal{G}$. If it is feasible to generate an appropriate secret-key set from an available group $\mathcal{G}$, then $\mathcal{G}$ is called a constructible group.

Signature structure. When signers sign a document sequentially, the signing order has a special meaning. We call such a signature structure, serial. On the other hand, when all or a part of the signers create a partial signature for a document in arbitrary order and a complete signature is created from these partial signatures, the signing order of the signers has no meaning. We call such a signature structure, parallel.

The signer group of a serial structure is denoted by $\left(P_{1}, P_{2}, \ldots, P_{n}\right)$. This means that $P_{1}$ signs first, $P_{2}$ second and so on. After all $P_{1}, P_{2}, \ldots, P_{n-1}$ have signed, $P_{n}$ signs. For simplicity, we write

$$
\begin{aligned}
G_{i, \ldots, j} & =\left(\ldots, P_{i-1}, P_{i}, \ldots, P_{j-1}, P_{j}, \ldots\right), \\
G_{j, \ldots, i} & =\left(\ldots, P_{i-1}, P_{j}, \ldots, P_{j-1}, P_{i}, \ldots\right),
\end{aligned}
$$

where $1 \leq i<j \leq n$.

The signer group of a parallel structure is denoted by $\wedge\left(P_{1}, P_{2}, \ldots, P_{n}\right)$.

In the scheme we propose the partial signature created by signer $P_{i}$ is a triple $\left(s_{i}, r_{i}, r\right) \in \mathrm{Z}_{q} \times \mathrm{Z}_{p}^{*} \times \mathrm{Z}_{p}^{*}$. The partial signature created by the last signer $P_{n}$ is the multisignature of the entire group. For $\left(s_{n}, r_{n}, r\right)$ we have $r_{n}=r$, so that the final form of the multisignature is $\left(s_{n}, r_{n}\right)$. 
Let $i, j \in\{1,2, \ldots, n\}, i<j$, and $K \subset\{1,2, \ldots, n\}$. For a variable $v$, let $v_{[i, j, K]}$ denote the sequence $v_{i}, v_{i+1}, \ldots, v_{j}$ in which all $v_{l}, l \in K$, are excluded. $v_{[1, n, \phi]}$ is simply denoted by $v_{[i, j]}$.

For simplicity, $(\bmod p)$ is sometimes omitted in this paper.

Hash function. In this paper we shall use a target-collision intractable hash function, hash, which takes values of arbitrary length as input and outputs a value in $\mathbf{Z}_{q}^{*}$. (A hash function is target-collision intractable if for a given $x$ it is infeasible to compute a $y \operatorname{such}$ that hash $(x)=\operatorname{hash}(y)$.)

\section{Attacks and Reductions}

Attack model. For our security analysis, we consider a model in which all insiders (signers) except an honest signer may collude in the attack. The attackers give a partial signature of a message $M$ to the honest signer $P_{i}$ and obtain a valid partial signature by $P_{i}$ of $M$. With this information, the attackers try to obtain forged signatures or to derive $P_{i}$ 's secret key.

Reductions. We use the following reductions in our security proof ( $c f$. [Wolls7]): $\circ f \leq_{m}^{p} g$ denotes that $f$ reduces to $g$ with respect to the polynomial-time many-one $\left(\leq_{m}^{p}\right)$ reducibility. (That is, there exists a polynomial-time computable function $h$ such that $f(x)=g(h(x))$.)

- $f \leq_{k-t t}^{p} g$ denotes that $f$ reduces to $g$ with respect to the polynomialtime truth-table $\left(\leq_{k-t t^{-}}^{p}\right)$ reducibility. In a $\leq_{k-t t^{p}}^{p}$-reducibility only $k$ nonadaptive queries to an oracle are allowed. If we do not wish to stress the number of queries, we simply write $f \leq_{t t}^{p} g$.

- $f \leq_{T}^{p} g$ denotes that $f$ reduces to $g$ with respect to the polynomialtime Turing $\left(\leq_{T^{-}}^{p}\right)$ reducibility. In a $\leq_{T}^{p}$-reducibility, a polynomial-time bounded oracle Turing machine can access an oracle adaptively.

For type $\in\{m, k-t t, T\}$, if $f \leq_{\text {type }}^{p} g$ and $g \leq_{\text {type }}^{p} f$, then $f \equiv_{\text {type }}^{p} g$.

We use the following functions for the proof of security.

- $\operatorname{DLP}_{q}(X, g, p, q)$ is a function that on input two primes $p, q$ with $q \mid(p-1)$, $X \in \mathrm{Z}_{p}^{*}, g \in \mathrm{Z}_{p}^{*}$ of order $q$, outputs $x \in \mathrm{Z}_{q}$ such that $X \equiv g^{x}(\bmod p)$, if such an $x$ exists. This function solves the discrete logarithm problem in a subgroup of prime order $q$.

- $\operatorname{Forge}(y, r, M, g, p, q)$ is a function that on input two primes $p, q$ with $q \mid(p-1), \quad y \in \mathrm{Z}_{p}^{*}, r \in \mathrm{Z}_{p}^{*}$ satisfying $\operatorname{gcd}(r, q)=1, M \in\{0,1\}^{*}, g \in \mathrm{Z}_{p}^{*}$ of order $q$, outputs $s^{\prime} \in \mathrm{Z}_{q}$ with $g^{s^{\prime}} \equiv y r^{r \cdot \operatorname{hash}(r, M)}(\bmod p)$ for a given hash function hash(), if such an $s^{\prime}$ exists.

This function forges a valid signature $s^{\prime}$ using the pair $(M, r)$ of a message $M$ and a number $r$ and available public information, but does not use the signer's secret key.

- $\operatorname{Secret1}(y, r, s, M, g, p, q)$ is a function that on input two primes $p, q$ with $q \mid(p-1), y \in \mathrm{Z}_{p}^{*}, r \in \mathrm{Z}_{p}^{*}$ with $\operatorname{gcd}(r, q)=1, s \in \mathrm{Z}_{q}, M \in\{0,1\}^{*}, g \in \mathrm{Z}_{p}^{*}$ of order $q$, outputs $(a, k) \in\left(\mathrm{Z}_{q}^{*}, \mathrm{Z}_{q}^{*}\right)$ such that 


$$
y \equiv g^{a}, r \equiv g^{k}, s \equiv a+k r \cdot \operatorname{hash}(r, M)(\bmod q)
$$

for a given hash function hash(), if such a pair of $(a, k)$ exists.

This function computes the secret key $a$ of $P$ and the random number $k$ using a valid signature $(r, s)$ by $P$ and available public information.

- Secret2 $\left[\left(P_{1}, P_{2}, \ldots, P_{n}\right)\right]\left(y_{i}, r_{i}, \tilde{r}, s_{i}, \tilde{k}, a_{[1, n,\{i\}]}, k_{[1, n,\{i\}]}, M, g, p, q\right)$ is a function that on input two primes $p, q$ with $q \mid(p-1), y_{i}, r_{i}, \in \mathrm{Z}_{p}^{*}, \tilde{r} \in \mathrm{Z}_{p}^{*}$ satisfying $\operatorname{gcd}(\tilde{r}, q)=1, s_{i}, \tilde{k} \in \mathrm{Z}_{q}, a_{[1, n,\{i\}]}, k_{[1, n,\{i\}]} \in \mathrm{Z}_{q}^{*} \times \cdots \times \mathrm{Z}_{q}^{*}$, $M \in\{0,1\}^{*}, g \in \mathrm{Z}_{p}^{*}$ of order $q$, outputs $\left(a_{i}, k_{i}\right) \in\left(\mathrm{Z}_{q}^{*}, \mathrm{Z}_{q}^{*}\right)$ such that $a_{[1, n]}$ is appropriate for $\left(P_{1}, P_{2}, \ldots, P_{n}\right)$, and

$$
\begin{aligned}
& \alpha=\left(\cdots\left(\left(a_{1}+1\right) a_{2}+1\right) \cdots\right) a_{i-1}(\bmod q), \\
& y_{i} \equiv g^{(\alpha+1) a_{i}}, r_{i} \equiv\left(g^{\tilde{k}}\right)^{a_{i}} \cdot g^{k_{i}}, \\
& s_{i} \equiv((\alpha+\tilde{k} \tilde{r} \cdot \operatorname{hash}(\tilde{r}, M))+1) a_{i}+k_{i} \tilde{r} \cdot \operatorname{hash}(\tilde{r}, M)(\bmod q),
\end{aligned}
$$

for a given hash function hash(), if such a pair of $\left(a_{i}, k_{i}\right)$ exists, and otherwise outputs $\perp$.

This function computes the pair $\left(a_{i}, k_{i}\right)$ consisting of the secret key $a_{i}$ and the random number $k_{i}$ of an honest signer $P_{i}$ in the following situation. All signers except $P_{i}$ collude to compute $\left(a_{i}, k_{i}\right)$, using any available public information and a valid partial signature $\left(r_{i}, s_{i}, r\right)$ created by $P_{i}$ in a serial structure for a message $M$. The colluding entities do not need to follow the signature creation procedure for computing these values. A partial signature $\left(\tilde{r}_{i-1}, \tilde{s}_{i-1}, \tilde{r}\right)$ given to $P_{i}$ satisfies $y_{i-1} \tilde{r}_{i-1}^{\tilde{r} \text { hash }(\tilde{r}, M)} \equiv g^{\alpha} \cdot g^{\tilde{k} \tilde{r} \cdot \operatorname{hash}(\tilde{r}, M)} \equiv g^{\tilde{s}_{i-1}}$, where $\tilde{r}_{i-1} \equiv g^{\tilde{k}}, \tilde{s}_{i-1} \equiv \alpha+\tilde{k} \tilde{r} \cdot \operatorname{hash}(\tilde{r}, M) \bmod q$.

- $\operatorname{Flip}\left[G_{i, \ldots, j}, G_{j, \ldots, i}\right]\left(y_{i-1}, y_{i}, y^{\prime}, \tilde{r}_{i-1}, r_{i}, \tilde{r}, \tilde{s}_{i-1}, s_{i}, \tilde{k}, a_{[1, n,\{i\}]}, k_{[1, n,\{i\}]}, M, g\right.$, $p, q)$ is a function that on input two primes $p, q$ with $q \mid(p-1), y_{i-1}, y_{i}, y^{\prime}$, $\tilde{r}_{i-1}, r_{i} \in \mathrm{Z}_{p}^{*}, \tilde{r} \in \mathrm{Z}_{p}^{*}$ satisfying $\operatorname{gcd}(\tilde{r}, q)=1, \tilde{s}_{i-1}, s_{i}, \tilde{k} \in \mathrm{Z}_{q}, a_{[1, n,\{i\}]}$, $k_{[1, n,\{i\}]} \in \mathrm{Z}_{q}^{*} \times \cdots \times \mathrm{Z}_{q}^{*}, M \in\{0,1\}^{*}, g \in \mathrm{Z}_{p}^{*}$ of order $q$, outputs $\left(s^{\prime}, r^{\prime}\right) \in\left(\mathrm{Z}_{q}, \mathrm{Z}_{p}^{*}\right)$ such that $s^{\prime} \in \mathrm{Z}_{q}, r^{\prime} \in \mathrm{Z}_{p}^{*}$ with $\operatorname{gcd}\left(r^{\prime}, q\right)=1, \alpha^{\prime} \in \mathrm{Z}_{q}^{*}$, $a_{i}, k_{i} \in \mathrm{Z}_{q}^{*}, a_{[1, n,\{i\}]}$ is appropriate for $\left[G_{i, \ldots, j}, G_{j, \ldots, i}\right]$, and

$$
\begin{aligned}
& \alpha=\left(\cdots\left(\left(a_{1}+1\right) a_{2}+1\right) \cdots\right) a_{i-1}(\bmod q), \\
& \beta= \alpha a_{j} a_{i+1} \cdots a_{j-1} a_{j+1} \cdots a_{n}+a_{j} a_{i+1} \cdots a_{j-1} a_{j+1} \cdots a_{n} \\
&+a_{i+1} \cdots a_{j-1} a_{j+1} \cdots a_{n}+\cdots \\
&+a_{j-1} a_{j+1} \cdots a_{n}+a_{j+1} \cdots a_{n}(\bmod q), \\
& \gamma= a_{j+1} \cdots a_{n}+\cdots+a_{n}(\bmod q), \\
& y_{i-1} \equiv g^{\alpha}, y_{i} \equiv\left(y_{i-1} \cdot g\right)^{a_{i}}, y^{\prime} \equiv g^{\beta a_{i}+\gamma}, \\
& \tilde{r}_{i-1} \equiv g^{\tilde{k}}, r_{i} \equiv \tilde{r}_{i-1}^{a_{i}} \cdot g^{k_{i}}, r^{\prime} \equiv g^{\alpha^{\prime}}, \\
& \tilde{s}_{i-1} \equiv \alpha+\tilde{k} \tilde{r} \cdot \operatorname{hash}(\tilde{r}, M)(\bmod q), \\
& s_{i} \equiv\left(\tilde{s}_{i-1}+1\right) a_{i}+k_{i} \tilde{r} \cdot \operatorname{hash}(\tilde{r}, M)(\bmod q), \\
& s^{\prime} \equiv\left(\beta a_{i}+\gamma\right)+\alpha^{\prime} r^{\prime} \cdot \operatorname{hash}\left(r^{\prime}, M\right)(\bmod q)
\end{aligned}
$$

for a given hash function hash(), if such a pair of $\left(s^{\prime}, r^{\prime}\right)$ exists.

This function forges a signature of a signer structure $G_{j, \ldots, i}$ from the signature of a signer structure $G_{i, \ldots, j}$ in the following situation. All signers except the honest signer $P_{i}$ collude in the forgery, using public information and a valid partial signature $\left(r_{i}, s_{i}, r\right)$ created by $P_{i}$ for the message $M$. The colluding entities do not need to follow the signature creation procedure for 
computing these values. A partial signature $\left(\tilde{r}_{i-1}, \tilde{s}_{i-1}, \tilde{r}\right)$ given to $P_{i}$ satisfies $y_{i-1} \tilde{r}_{i-1}^{\tilde{r} \operatorname{hash}(\tilde{r}, M)} \equiv g^{\alpha} \cdot g^{\tilde{k} \tilde{r} \cdot \operatorname{hash}(\tilde{r}, M)} \equiv g^{\tilde{s}_{i-1}}$, where $\tilde{r}_{i-1} \equiv g^{\tilde{k}}, \tilde{s}_{i-1} \equiv$ $\alpha+\tilde{k} \tilde{r} \cdot \operatorname{hash}(\tilde{r}, M)(\bmod q)$.

- Forge $\left[G_{o}, G_{f}\right]\left(y_{i-1}, y_{i}, y^{\prime}, \tilde{r}_{i-1}, r_{i}, \tilde{r}, \tilde{s}_{i-1}, s_{i}, \tilde{\beta}, a_{[1, n,\{i\}]}, k_{[1, n,\{i\}]}, M, g, p, q\right)$ is a function that on input two primes $p, q$ with $q \mid(p-1), y_{i-1}, y_{i}, y^{\prime} \in \mathrm{Z}_{p}^{*}$, $\tilde{r}_{i-1} \in \mathrm{Z}_{p}^{*}$ satisfying $\operatorname{gcd}\left(\tilde{r}_{i-1}, q\right)=1, r_{i} \in \mathrm{Z}_{p}^{*}$ satisfying $\operatorname{gcd}\left(r_{i}, q\right)=1$, $\tilde{s}_{i-1}, s_{i} \in \mathrm{Z}_{q}, \tilde{\beta} \in \mathrm{Z}_{q}^{*}, a_{[1, n,\{i\}]}, k_{[1, n,\{i\}]} \in \mathrm{Z}_{q}^{*} \times \cdots \times \mathrm{Z}_{q}^{*}, M \in\{0,1\}^{*}, g \in \mathrm{Z}_{p}^{*}$ of order $q$, outputs $\left(s^{\prime}, r^{\prime}\right) \in\left(\mathrm{Z}_{q}, \mathrm{Z}_{p}^{*}\right)$ such that $s^{\prime} \in \mathrm{Z}_{q}, r^{\prime} \in \mathrm{Z}_{p}^{*}$ satisfying $\operatorname{gcd}\left(r^{\prime}, q\right)=1, \beta^{\prime} \in \mathrm{Z}_{q}, a_{i}, k_{i} \in \mathrm{Z}_{q}^{*}, a_{[1, n]}$ is appropriate for $\mathcal{G} \subset S P G$, function $f_{\alpha}$ is determined from $G_{o} \in \mathcal{G}, f_{\alpha_{1}^{\prime}}$ and $f_{\alpha_{2}^{\prime}}$ are determined from $G_{f} \in \mathcal{G}, P_{i} \in G_{o}, P_{i} \in G_{f}$, and

$$
\begin{aligned}
& y_{i-1} \equiv g^{\alpha}, y_{i} \equiv\left(y_{i-1} \cdot g\right)^{a_{i}}, y^{\prime} \equiv g^{\alpha_{1}^{\prime} a_{i}+\alpha_{2}^{\prime}}, \\
& \alpha=f_{\alpha}\left(a_{[1, n,\{i\}]}\right), \alpha_{1}^{\prime}=f_{\alpha_{1}^{\prime}}\left(a_{[1, n,\{i\}]}\right), \alpha_{2}^{\prime}=f_{\alpha_{2}^{\prime}}\left(a_{[1, n,\{i\}]}\right), \\
& \tilde{r}_{i-1} \equiv g^{\tilde{\beta}}, r_{i} \equiv \tilde{r}_{i-1}^{a_{i}} \cdot g^{k_{i}}, r^{\prime} \equiv g^{\beta^{\prime}}, \\
& \tilde{s}_{i-1} \equiv \alpha+\tilde{\beta} \tilde{r} \operatorname{hash}(\tilde{r}, M)(\bmod q), \\
& s_{i} \equiv\left(\tilde{s}_{i-1}+1\right) a_{i}+k_{i} \tilde{r} \operatorname{hash}(\tilde{r}, M)(\bmod q), \\
& s^{\prime} \equiv \alpha_{1}^{\prime} a_{i}+\alpha_{2}^{\prime}+\beta^{\prime} r^{\prime} \cdot \operatorname{hash}\left(r^{\prime}, M\right)(\bmod q),
\end{aligned}
$$

for a given hash function hash( $)$, if such a pair of $\left(s^{\prime}, r^{\prime}\right)$ exists.

This function forges the signature of a signer structure $G_{f}$ from the signature of a signer structure $G_{o}$ in the following situation. All signers except the honest signer $P_{i}$ collude in the forgery using public information and a valid partial signature $\left(r_{i}, s_{i}, r\right)$ created by $P_{i}$ for the message $M$. The colluding entities do not need to follow the signature creation procedure for computing these values. A partial signature $\left(\tilde{r}_{i-1}, \tilde{s}_{i-1}, \tilde{r}\right)$ given to $P_{i}$ satisfies $y_{i-1} \tilde{r}_{i-1}^{\tilde{r} \operatorname{hash}(\tilde{r}, M)} \equiv g^{\alpha} \cdot g^{\tilde{\beta} \tilde{r} \cdot \operatorname{hash}(\tilde{r}, M)} \equiv g^{\tilde{s}_{i-1}}$, where $\tilde{r}_{i-1} \equiv g^{\tilde{\beta}}, \tilde{s}_{i-1} \equiv$ $\alpha+\tilde{\beta} \tilde{r} \cdot \operatorname{hash}(\tilde{r}, M)(\bmod q)$.

- $F C(v, w)$ is a function which on input $v, w$, outputs the first component $v$.

\section{Modified ElGamal Signature}

We use a modified version [YL93] of the ElGamal signature scheme [ElG85]. To explain this scheme we consider a single entity signer group consisting of only $P$. The message is $M$. Let $a \in \mathrm{Z}_{q}^{*}$ be the secret key and $y=g^{a}(\bmod p)$ the public key of $P$.

\section{Basic signature protocol}

1. $P$ selects $k \in_{R} \mathrm{Z}_{q}^{*}$ and computes $r=g^{k} \bmod p$. $P$ repeats this process until $r$ satisfies $\operatorname{gcd}(r, q)=1$.

2. $P$ computes $s=a+k r \cdot \operatorname{hash}(r, M)(\bmod q)$. The signature of $M$ is $(s, r)$.

3. The signature $(s, r)$ is verified by checking that $g^{s} \stackrel{?}{\equiv} y r^{r \cdot \operatorname{hash}(r, M)}(\bmod p)$.

A valid signature passes the verification check because

$$
g^{s} \equiv g^{a} \cdot g^{k r \cdot \operatorname{hash}(r, M)} \equiv y r^{r \cdot \operatorname{hash}(r, M)}(\bmod p)
$$




\subsection{Security Considerations}

Generation of a forged signature $(r, s)$ : To show the difficulty of forging a signature we first consider the case when $r$ is given.

- Forge $\leq_{m}^{p} \mathbf{D L P}_{q}: \operatorname{Forge}(y, r, M, g, p, q)=\mathbf{D L P}_{q}\left(y r^{r \cdot h a s h}(r, M), g, p, q\right)$

- $\mathbf{D L P}_{q} \leq_{m}^{p}$ Forge: $\operatorname{DLP}_{q}\left(g^{s}, g, p, q\right)=\operatorname{Forge}(y, r, M, g, p, q)$, where $r \in_{R} \mathrm{Z}_{p}^{*}, \operatorname{gcd}(r, q)=1, M \in_{R}\{0,1\}^{*}, y=g^{s} \cdot r^{-r \cdot h a s h}(r, M)$.

Observe that

$$
y \cdot r^{r \cdot \operatorname{hash}(r, M)} \equiv\left(g^{s} \cdot r^{-r \cdot \operatorname{hash}(r, M)}\right) \cdot r^{r \cdot \operatorname{hash}(r, M)} \equiv g^{s} .
$$

Thus $\operatorname{DLP}_{q} \equiv{ }_{m}^{p}$ Forge.

This proof shows only one aspect of the difficulty of signature forgery.

\section{Security of secret values}

- Secret1 $\leq_{2-t t}^{p} \operatorname{DLP}_{q}$ :

$\operatorname{Secret1}(y, r, s, M, g, p, q)=\left(\mathbf{D L P}_{q}(y, g, p, q), \mathbf{D L P}_{q}(r, g, p, q)\right)$

- $\mathbf{D L P}_{q} \leq_{1-t t}^{p}$ Secret1: $\operatorname{DLP}_{q}\left(g^{a}, g, p, q\right)=(r \cdot \operatorname{hash}(r, M))^{-1}$.

$\cdot(-s(1-r \cdot \operatorname{hash}(r, M))+F C(\operatorname{Secret1}(y, r, s, M, g, p, q)))(\bmod q)$,

where $s \in_{R} \mathrm{Z}_{q}^{*}, M \in_{R}\{0,1\}^{*}, r=\left(g^{a}\right)^{-1} \cdot g^{s}, \operatorname{gcd}(r \cdot \operatorname{hash}(r, M), q)=1$, and $y=g^{s} \cdot r^{-r \cdot h a s h(r, M)}$.

Observe that

$$
y \cdot r^{r \cdot h a s h(r, M)}=g^{s}
$$

and that

$$
\begin{aligned}
y & \equiv g^{s} \cdot r^{-r \cdot h a s h}(r, M) \equiv g^{s} \cdot g^{(-a+s)(-r \operatorname{hash}(r, M))} \\
& \equiv g^{s(1-r \operatorname{hash}(r, M))+\operatorname{ar} \cdot \operatorname{hash}(r, M)} .
\end{aligned}
$$

Thus $\operatorname{DLP}_{q} \equiv_{t t}^{p}$ Secret1.

\section{Serial Multisignature Scheme}

A partial public key $y_{i}$ of $P_{i}$ of a multisignature scheme must belong to $Z_{p}^{*} \backslash\{1\}$ and must also satisfy the condition (1). This is achieved by selecting appropriate secret-key groups.

For example, an appropriate secret-key group $\left(a_{1}, \ldots, a_{n}\right)$ for $\left(P_{1}, \ldots, P_{n}\right)$ is obtained by choosing $\left(\alpha_{i-1}+1\right) a_{i} \neq a_{j}$ for $i=2, \ldots, n, j=1, \ldots, i-1$, where $\alpha_{i-1}=\left(\ldots\left(\left(a_{1}+1\right) a_{2}+1\right) \ldots\right) a_{i-1}(\bmod q) \quad($ See Section 7.2).

Suppose $n$ signers $P_{i}$ sign a message $M$ sequentially. Let $a_{i} \in \mathrm{Z}_{q}^{*}$ be the secret key of $P_{i}$. Then the partial public key $y_{i}$ of $P_{i}$ in $\left(P_{1}, \ldots, P_{n}\right), i=1,2, \ldots, n$, is computed as follows:

$$
y_{1}=g^{a_{1}}, y_{i}=\left(y_{i-1} \cdot g\right)^{a_{i}} .
$$

The public key of the group $\left(P_{1}, \ldots, P_{n}\right)$ is $y=y_{n}(\bmod p)$. The secret and public keys are generated either by a trusted center or by each signer using distributed 
protocols. In the latter case, each signer repeatedly selects his secret key until the keys form an appropriate set. Furthermore, each signer needs to prove that they know the secret key which corresponds to the partial public key computed. For this purpose they may use the protocol in [Schn91], which does not reveal any secret information.

\section{Serial multisignature protocol}

1. Generation of $r$. $P_{1}, \ldots, P_{n}$ generate $r$ together as follows.

(a) $P_{1}$ selects $k_{1} \in_{R} \mathrm{Z}_{q}^{*}$ and computes $r_{1}=g^{k_{1}} \bmod p$. $P_{1}$ repeats this process until $r_{1}$ satisfies $\operatorname{gcd}\left(r_{1}, q\right)=1$.

(b) For $i \in\{2, \ldots, n\}$ :

$P_{i-1}$ gives $r_{i-1}$ to $P_{i}$.

$P_{i}$ selects $k_{i} \in_{R} \mathrm{Z}_{q}^{*}$ and computes $r_{i}=r_{i-1}{ }^{a_{i}} g^{k_{i}} \bmod p ; P_{i}$ repeats this process until $r_{i}$ satisfies $\operatorname{gcd}\left(r_{i}, q\right)=1$.

(c) $r=r_{n}$.

2. Generation of $s . P_{1}, \ldots, P_{n}$ generate $s$ together as follows.

(a) $P_{1}$ computes $s_{1}=a_{1}+k_{1} r \cdot \operatorname{hash}(r, M)(\bmod q)$.

(b) For $i \in\{2, \ldots, n\}$ :

$P_{i-1}$ gives $s_{i-1}$ to $P_{i}$.

$P_{i}$ verifies that $g^{s_{i-1}} \equiv y_{i-1} r_{i-1}^{r \cdot h a s h}(r, M)(\bmod p)$ and if so computes

$$
s_{i}=\left(s_{i-1}+1\right) a_{i}+k_{i} r \cdot \operatorname{hash}(r, M)(\bmod q) .
$$

(c) $s=s_{n}$.

Multisignature. $(r, s)$ is the multisignature on $M$ by $\left(P_{1}, \ldots, P_{n}\right)$.

Verification. A multisignature $(r, s)$ is verified by checking the congruence

$$
g^{s} \stackrel{?}{\equiv} y r^{r \cdot h a s h}(r, M)(\bmod p) \text {. }
$$

\subsection{Security Considerations}

Security of $a_{i}$ and $k_{i}$. We consider the case when all the signers $P_{j}$ except an honest signer $P_{i}, i>1$, collude to derive $a_{i}$ and $k_{i}$ from any information they can obtain.

Let $\alpha$ be the exponent of $y_{i-1}$ to the base $g$ modulo $p$, i.e. $y_{i-1}=g^{\alpha}, \alpha=$ $\left(\ldots\left(\left(a_{1}+1\right) a_{2}+1\right) \ldots\right) a_{i-1}(\bmod q)$. Likewise, let $\tilde{k}$ be the exponent of $\tilde{r}_{i-1}$ to the base $g$ modulo $p$, i.e. $\tilde{r}_{i-1}=g^{\tilde{k}}$.

- Secret2 $\left[\left(P_{1}, \ldots, P_{i}, \ldots, P_{n}\right)\right] \leq_{T}^{p} \operatorname{DLP}_{q}:$

Secret2 $\left[\left(P_{1}, \ldots, P_{i}, \ldots, P_{n}\right)\right]\left(y_{i}, r_{i}, \tilde{r}, s_{i}, \tilde{k}, a_{[1, n,\{i\}]} k_{[1, n,\{i\}]}, M, g, p, q\right)=$ $\left(A, \mathbf{D L P}_{q}\left(r_{i} \cdot\left(g^{-A \tilde{k}}\right), g, p, q\right)\right)$, where $A=\mathbf{D L P}_{q}\left(y_{i}, g^{\alpha+1}, p, q\right)$. 
- $\mathbf{D L P}_{q} \leq_{m}^{p} \operatorname{Secret2}\left[\left(P_{1}, \ldots, P_{i}, \ldots, P_{n}\right)\right]$ :

At first $a_{[1, i-1]} \in \mathrm{Z}_{q}^{*} \times \cdots \times \mathrm{Z}_{q}^{*}$ is selected so that it is appropriate for $\left(P_{1}, \ldots, P_{i}, \ldots, P_{i-1}\right)$. Then $a_{[i+1, n]} \in R \mathrm{Z}_{q}^{*} \times \cdots \times \mathrm{Z}_{q}^{*}$ is randomly selected. This procedure is repeated until the set of keys $a_{[1, n,\{i\}]}$ and $a_{i}$ becomes appropriate for $\left(P_{1}, \ldots, P_{i}, \ldots, P_{n}\right)$. We can recognize that $a_{[1, n]}$ is not appropriate from the output of Secret2 $\left[\left(P_{1}, \ldots, P_{i}, \ldots, P_{n}\right)\right]$. In Section 7.2 we shall see that this procedure is feasible (with probability almost one) if $n$ is bounded by a polynomial in $|p|$. When $a_{[1, n]}$ becomes appropriate, $\mathbf{D L P}_{q}\left(g^{a_{i}}, g, p, q\right)$ is computed in the following way:

$\operatorname{DLP}_{q}\left(g^{a_{i}}, g, p, q\right)=F C\left(\right.$ Secret2 $\left[\left(P_{1}, \ldots, P_{i}, \ldots, P_{n}\right)\right]\left(\left(g^{a_{i}}\right)^{\alpha+1},\left(g^{a_{i}}\right)^{\tilde{k}} \cdot g^{k_{i}}\right.$, $\left.\left.\tilde{r}, k_{i} \tilde{r} \cdot \operatorname{hash}(\tilde{r}, M)(\bmod q), \tilde{k}, a_{[1, n,\{i\}]}, k_{[1, n,\{i\}]}, M, g, p, q\right)\right)$,

where $\tilde{r} \in_{R} \mathrm{Z}_{p}^{*}, \operatorname{gcd}(\tilde{r}, q)=1, k_{i} \in_{R} \mathrm{Z}_{q}^{*}, M \in_{R}\{0,1\}^{*}, a_{[1, n]}$ is appropriate for $\left(P_{1}, \ldots, P_{i}, \ldots, P_{n}\right), a_{[1, n,\{i\}]} \in \mathrm{Z}_{q}^{*} \times \cdots \times \mathrm{Z}_{q}^{*}, k_{[1, n,\{i\}]} \in \in_{R} \mathrm{Z}_{q}^{*} \times \cdots \times \mathrm{Z}_{q}^{*}$, $\alpha \in \mathrm{Z}_{q}^{*} \backslash\{q-1\}, \tilde{k} \in \mathrm{Z}_{q}^{*} \backslash\{1\}$,

$\alpha=\left(\ldots\left(\left(a_{1}+1\right) a_{2}+1\right) \ldots\right) a_{i-1}(\bmod q)$,

$\tilde{k}=-(\alpha+1)(\tilde{r} \cdot \operatorname{hash}(\tilde{r}, M))^{-1}(\bmod q)$.

Therefore $\mathbf{D L P}_{q} \equiv_{T}^{p} \operatorname{Secret2}\left[\left(P_{1}, \ldots, P_{i}, \ldots, P_{n}\right)\right]$.

Security of Signing Order. We study the order of signature creation. Namely we assess the difficulty of forgery when all attackers $P_{k} \in G_{i, \ldots, j}$ excluding $P_{i} \operatorname{try}$ to collude to change the signing order from $G_{i, \ldots, j}$ to $G_{j, \ldots, i}$, where $1 \leq i<j \leq n$, after $P_{i}$, who is given a possibly forged partial signature from the previous signer, has signed $M$. Attackers can use any information except $a_{i}$ and $k_{i}$.

Let $y^{\prime}$ be a public key of signature structure $\left(\ldots, P_{i-1}, P_{j}, \ldots, P_{i}, \ldots\right)$ and $\left(r^{\prime}, s^{\prime}\right)$ be a forged signature. $\tilde{k}$ and $\tilde{r}$ are freely selected by attackers.

- $\operatorname{Flip}\left[G_{i, \ldots, j}, G_{j, \ldots, i}\right] \leq_{1-t t}^{p} \operatorname{DLP}_{q}:$

$\operatorname{Flip}\left[G_{i, \ldots, j}, G_{j, \ldots, i}\right]\left(y_{i-1}, y_{i}, y^{\prime}, \tilde{r}_{i-1}, r_{i}, \tilde{r}, \tilde{s}_{i-1}, s_{i}, \tilde{k}, a_{[1, n,\{i\}]}, k_{[1, n,\{i\}]}\right.$,

$M, g, p, q)=\left(\mathbf{D L P}_{q}\left(y^{\prime}, g, p, q\right)+\alpha^{\prime} r^{\prime} \cdot \operatorname{hash}\left(r^{\prime}, M\right)(\bmod q), r^{\prime}\right)$,

where $\alpha^{\prime} \in_{R} \mathrm{Z}_{q}^{*}, r^{\prime}=g^{\alpha^{\prime}}, \operatorname{gcd}\left(r^{\prime}, q\right)=1$.

- $\mathbf{D L P}_{q} \leq_{1-t t}^{p} \mathbf{F l i p}\left[G_{i, \ldots, j}, G_{j, \ldots, i}\right]$ :

$\operatorname{DLP}\left(g^{a_{i}}, g, p, q\right)=\beta^{-1} \cdot\left\{-\gamma-\alpha^{\prime} r^{\prime} \cdot \operatorname{hash}\left(r^{\prime}, M\right)+F C\left(\mathbf{F l i p}\left[G_{i, \ldots, j}, G_{j, \ldots, i}\right]\right.\right.$ $\left(g^{\alpha},\left(g^{a_{i}}\right)^{\alpha+1},\left(g^{a_{i}}\right)^{\beta} \cdot g^{\gamma}, g^{\tilde{k}},\left(g^{s_{i}} \cdot\left(g^{a_{i}}\right)^{-(\alpha+1)}\right)^{(\tilde{r} \cdot \operatorname{hash}(\tilde{r}, M))^{-1}}, \tilde{r}, \alpha+\tilde{k} \tilde{r} \cdot \operatorname{hash}(\tilde{r}, M)\right.$, $\left.\left.\left.s_{i}, \tilde{k}, a_{[1, n,\{i\}]}, k_{[1, n,\{i\}]}, M, g, p, q\right)\right)\right\}(\bmod q)$,

where $\tilde{k} \in_{R} \mathrm{Z}_{q}^{*}, s_{i} \in_{R} \mathrm{Z}_{q}, \tilde{r} \in_{R} \mathrm{Z}_{q}^{*}$ with $\operatorname{gcd}(\tilde{r}, q)=1, a_{[1, n,\{i\}]} \in \mathrm{Z}_{q}^{*} \times \cdots \times \mathrm{Z}_{q}^{*}$ is appropriate for $\left\{G_{i, \ldots, j}, G_{j, \ldots, i}\right\}, k_{[1, n,\{i\}]} \in_{R} \mathrm{Z}_{q}^{*} \times \cdots \times \mathrm{Z}_{q}^{*}, M \in_{R}\{0,1\}^{*}$, $\alpha^{\prime} \in_{R} \mathrm{Z}_{q}^{*}, r^{\prime}=g^{\alpha^{\prime}}, \operatorname{gcd}\left(r^{\prime} q\right)=1$,

$$
\begin{aligned}
\alpha= & \left(\cdots\left(\left(a_{1}+1\right) a_{2}+1\right) \cdots\right) a_{i-1}(\bmod q), \\
\beta= & \alpha a_{i+1} \cdots a_{n}+a_{i+1} \cdots a_{n}+a_{i+1} \cdots a_{j-1} a_{j+1} \cdots a_{n}+\cdots+ \\
& +a_{j-1} a_{j+1} \cdots a_{n}+a_{j+1} \cdots a_{n}(\bmod q), \operatorname{gcd}(\beta, q)=1, \\
\gamma= & a_{j+1} \cdots a_{n}+\cdots+a_{n}(\bmod q) .
\end{aligned}
$$

Therefore $\mathbf{D L P}_{q} \equiv_{1-t t}^{p} \operatorname{Flip}\left[G_{i, \ldots, j}, G_{j, \ldots, i}\right]$. 


\subsection{Appropriate Secret Keys}

Given $a_{i} \in \mathrm{Z}_{q}^{*}$ select $a_{[i+1, n]} \in \in_{R} \mathrm{Z}_{q}^{*} \times \cdots \times \mathrm{Z}_{q}^{*}$ after selecting $a_{[1, i-1]}$ appropriately for $\left(P_{1}, \ldots, P_{i}\right)$. The probability that $a_{[1, n]}$ is appropriate for $\left(P_{1}, \ldots, P_{i}, \ldots, P_{n}\right)$ can be estimated as follows.

Let $\alpha_{i-1}=\left(\cdots\left(\left(a_{1}+1\right) a_{2}+1\right) \cdots\right) a_{i-1}(\bmod q)$. Then $a_{i}$ should satisfy

$$
a_{i} \neq \alpha_{j}\left(\alpha_{i-1}+1\right)^{-1}(\bmod q) \text { for } j=1, \ldots, i-1
$$

and

$$
a_{i} \neq\left(a_{u}-\sum_{l=i+1} \prod_{t=l}^{m} a_{t}\right)\left(\alpha_{i-1}+1\right)^{-1}\left(\prod_{k=i+1}^{m} a_{k}\right)^{-1}(\bmod q)
$$

for $u=1, \ldots, m-1$ and $m=i+1, \ldots, n$. Thus the probability is

$$
1-\left(i-1+\sum_{v=i}^{n-1} v\right) / 2^{\left|a_{i}\right|}=1-\left(n(n-1)-i^{2}+3 i-2\right) / 2^{\left|a_{i}\right|-1}
$$

for $i=2, \ldots, n$. If $n=O\left(\operatorname{poly}\left(\left|a_{i}\right|\right)\right)$ this probability can be estimated to be almost 1 .

\section{Parallel Multisignature Scheme}

The public key of a parallel multisignature scheme is $y=\prod_{i=1}^{n} y_{i}(\bmod p)$, where $y_{i}=g^{a_{i}}$ are the partial public keys. We must have $y_{i} \in \mathrm{Z}_{p}^{*} \backslash\{1\}$ and the product of any partial keys should not be 1 .

\section{Parallel multisignature protocol}

1. $P_{i}$ selects $k_{i} \in_{R} \mathrm{Z}_{q}^{*}$ and computes $r_{i}=g^{k_{i}}(\bmod p) . P_{i}$ repeats this process until $\operatorname{gcd}\left(r_{i}, q\right)=1$

2. Each $P_{i}$ broadcasts $r_{i}$ to all the other signers. Then each $P_{i}$ checks if there is a combination of $r_{i}$ whose product is equal to 1 modulo $p$. If there is such a combination, step 1 is repeated.

3. $r=\prod_{i=1}^{n} r_{i}(\bmod p)$.

4. $P_{i}$ computes $s_{i}=a_{i}+k_{i} r \cdot \operatorname{hash}(r, M)(\bmod q)$, and broadcasts $s_{i}$ to all the other signers.

5. $s=\sum_{i=1}^{n} s_{i}(\bmod q)$.

The multisignature of $M$ by $\wedge\left(P_{1}, \ldots, P_{n}\right)$ is $(s, r)$. It is verified by checking that

$$
g^{s} \equiv g^{\sum_{i=1}^{n} s_{i}} \equiv \prod_{i=1}^{n} y_{i}\left(\prod_{i=1}^{n} r_{i}\right)^{r \cdot \operatorname{hash}(r, M)} \equiv y r^{r \cdot \operatorname{hash}(r, M)}(\bmod p) .
$$


Observe that,

$$
\begin{aligned}
g^{s} & \equiv g^{\sum_{i=1}^{n} s_{i}} \equiv \prod_{i=1}^{n} y_{i}\left(\prod_{i=1}^{n} r_{i}\right)^{r \cdot \operatorname{hash}(r, M)} \\
& \equiv y r^{r \cdot \operatorname{hash}(r, M)}(\bmod p)
\end{aligned}
$$

As noted in [DOMU94,DOM98] parallel multisignatures schemes are essentially threshold schemes [DF91].

\section{Multisignature for a Mixed Structure}

The parallel and serial signature structures can be combined in an arbitrary order. The public keys and signatures are generated and verified by using the methods of the serial and parallel cases in a straightforward way.

The partial public keys of the signers in a mixed structure are computed as follows. Let $G_{\text {init }}$ be the group of signers with no incoming edges in the graph representation $G$. The partial public key of signer $P_{i}$ in $G_{\text {init }}$ is simply $y_{i}=g^{a_{i}}$. This is given to every signer in $G_{n e x t, \text { init }}$, the group of signers whose edges are connected to the edges of $G_{\text {init }}$. Then for all $i$ such that $P_{i}$ does not belong to $G_{\text {init }}, P_{i}$ 's partial public key is $y_{i}=\left(g \prod_{j: P_{j} \in G_{p r e v, i}} y_{j}\right)^{a_{i}}$ and is given to every signer in $G_{n e x t, i}$, where $G_{p r e v, i}$ and $G_{n e x t, i}$ denote a group of signers whose edges are connected to and from $P_{i}$ in the graph representation, respectively. The public key of the group is $y=\prod_{j: P_{j} \in G_{\text {last }}} y_{j}$, where $G_{\text {last }}$ denotes a group of signers whose edges are combined into the output in the graph representation.

\section{General multisignature protocol}

1. Computing $r$ : For all $P_{i}$ which belong to $G_{\text {init }}, r_{i}=g^{k_{i}}$, with $k_{i} \in_{R} \mathrm{Z}_{q}^{*}$. For all $P_{i}$ that do not belong to $G_{i n i t}, r_{i}=\left(\prod_{j: P_{j} \in G_{p r e v, i}} r_{j}\right)^{a_{i}} g^{k_{i}}$ where $k_{i} \in R \mathrm{Z}_{q}^{*}$. The created $r_{i}$ is given to every signer in $G_{n e x t, i}$. Then $r=$ $\prod_{j: P_{j} \in G_{\text {last }}} r_{j}$.

2. Computing $s$ : For all $P_{i}$ which belong to $G_{\text {init }}$, a partial signature $\left(s_{i}, r_{i}, r\right)$ of $P_{i}$ is computed by $s_{i}=a_{i}+k_{i} r \cdot \operatorname{hash}(r, M)(\bmod q)$. Then for all $P_{i}$ that do not belong to $G_{\text {init }}$, the partial signature $\left(s_{i}, r_{i}, r\right)$ of $P_{i}$ is computed by $s_{i}=\left(\left(\sum_{j: P_{j} \in G_{p r e v, i}} s_{j}\right)+1\right) a_{i}+k_{i} r \cdot \operatorname{hash}(r, M)(\bmod q)$, and given to every signer in $G_{n e x t, i}$. Finally $s=\sum_{j: P_{j} \in G_{\text {last }}} s_{j}$.

The multisignature of $M$ by the group $G$ is $(s, r)$. It is verified by checking that $g^{s} \stackrel{?}{\equiv} y r^{r \cdot h a s h}(r, M)(\bmod p)$.

To show how this is done more explicitly we compute the multisignature of the group $G_{f i g 1}=\left[\wedge\left(P_{1},\left(P_{2}, P_{3}, P_{4}\right)\right), \wedge\left(\left(P_{5}, P_{6}\right), P_{7},\left(P_{8}, P_{9}\right)\right)\right]$ in Figure 1. The partial public key of $P_{i}$ in $G_{f i g 1}$ is computed from the secret key $a_{i} \in \mathrm{Z}_{q}^{*}$ and other partial keys $y_{j}(j \neq i)$ as follows: $y_{1}=g^{a_{1}}(\bmod p)$ for the first 
lower branch, $y_{2}=g^{a_{2}}(\bmod p), y_{3}=\left(y_{2} g\right)^{a_{3}}(\bmod p), y_{4}=\left(y_{3} g\right)^{a_{4}}(\bmod p)$ for the first upper branch, $y_{5}=\left(\left(y_{1} y_{4}\right) g\right)^{a_{5}}(\bmod p), y_{6}=\left(y_{5} g\right)^{a_{6}}(\bmod p)$ for the second lower branch, $y_{7}=\left(\left(y_{1} y_{4}\right) g\right)^{a_{7}}(\bmod p)$ for the second middle branch, $y_{8}=\left(\left(y_{1} y_{4}\right) g\right)^{a_{8}}(\bmod p), y_{9}=\left(y_{8} g\right)^{a_{9}}(\bmod p)$ for the second upper branch. Finally the public key $y$ of $G_{f i g 1}$ is computed by $y=y_{6} y_{7} y_{9}(\bmod p)$.

- Computing $r$ : We have $r_{4}=r_{3}^{a_{4}} g^{k_{4}}(\bmod p), r_{5}=\left(r_{1} r_{4}\right)^{a_{5}} g^{k_{5}}(\bmod p), r_{6}=$ $\left(r_{5}\right)^{a_{6}} g^{k_{6}}(\bmod p)$, so $r=r_{6} r_{7} r_{9}(\bmod p)$.

- Computing $s$ : We have $s_{4}=\left(s_{3}+1\right) a_{4}+k_{4} r \cdot \operatorname{hash}(r, M)(\bmod q), s_{5}=$ $\left(\left(s_{1}+s_{4}\right)+1\right) a_{5}+k_{5} r \cdot h \operatorname{hash}(r, M)(\bmod q)$ and $s_{6}=\left(s_{5}+1\right) a_{6}+$ $k_{6} r \cdot \operatorname{hash}(r, M)(\bmod q)$, so $s=s_{6}+s_{7}+s_{9}(\bmod q)$.

The multisignature of $M$ by $G_{f i g 1}$ is $(s, r)$. It is verified by checking that

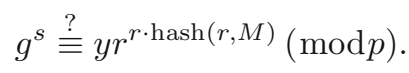

\subsection{Security Considerations}

The partial public key of a signer $P_{i}$ in a directed series-parallel graph can be expressed as $y=g^{A a_{i}+B}$, for some $A, B \in Z_{q}$. We study the difficulty of signature forgery for a structure $G_{f}$, given a partial signature $\left(r_{i}, s_{i}\right)$ of $P_{i}$ for the original structure $G_{0}$.

- Forge $\left[G_{o}, G_{f}\right] \leq_{1-t t}^{p} \mathbf{D L P}_{q}:$

Forge $\left[G_{o}, G_{f}\right]\left(y_{i-1}, y_{i}, y^{\prime}, \tilde{r}_{i-1}, r_{i}, \tilde{r}, \tilde{s}_{i-1}, s_{i}, \tilde{\beta}, a_{[1, n,\{i\}]}, k_{[1, n,\{i\}]}, M, g, p, q\right)$ $=\left(\mathbf{D L P}\left(y^{\prime}, g, p, q\right)+\beta^{\prime} r^{\prime} \operatorname{hash}\left(r^{\prime}, M\right)(\bmod q), r^{\prime}\right)$, where $\beta^{\prime} \in_{R} \mathbf{Z}_{q}^{*}, r^{\prime}=g^{\beta^{\prime}}$, $\operatorname{gcd}\left(g^{\beta^{\prime}}, q\right)=1$.

- $\mathbf{D L P}_{q} \leq_{1-t t}^{p}$ Forge $\left[G_{o}, G_{f}\right]$ :

$\operatorname{DLP}_{q}\left(g^{a_{i}}, g, p, q\right)=\alpha_{1}^{\prime-1}\left\{-\beta^{\prime} r^{\prime} \operatorname{hash}\left(r^{\prime}, M\right)-\alpha_{2}^{\prime}+F C\left(\right.\right.$ Forge $\left[G_{o}, G_{f}\right]\left(g^{\alpha}\right.$, $\left.\left(g^{a_{i}}\right)^{\alpha+1},\left(g^{a_{i}}\right)^{\alpha_{1}^{\prime}} \cdot g^{\alpha_{2}^{\prime}}, g^{\tilde{\beta}},\left(g^{s_{i}} \cdot\left(g^{a_{i}}\right)^{-\alpha-1}\right)^{(\tilde{r} \cdot h a s h}(\tilde{r}, M)\right)^{-1}, \tilde{r}, \alpha+\tilde{\beta} \tilde{r}$. $\left.\left.\left.\operatorname{hash}(\tilde{r}, M), s_{i}, \tilde{\beta}, a_{[1, n,\{i\}]}, k_{[1, n,\{i\}]}, M, g, p, q\right)\right)\right\}(\bmod q)$,

where $a_{[1, n]} \in \mathrm{Z}_{q}^{*} \times \cdots \times \mathrm{Z}_{q}^{*}$ is appropriate for $\left\{G_{o}, G_{f}\right\}, G_{o}, G_{f} \in \mathcal{G}, \tilde{\beta} \in_{R} \mathrm{Z}_{q}^{*}$ with $\operatorname{gcd}\left(g^{\tilde{\beta}}, q\right)=1, s_{i} \in_{R} \mathrm{Z}_{q}$ with $\operatorname{gcd}\left(\left(g^{s_{i}} \cdot\left(g^{a_{i}}\right)^{-\alpha-1}\right)^{(\tilde{r} \operatorname{hash}(\tilde{r}, M))^{-1}}, q\right)=1$, $\tilde{r} \in_{R} \mathrm{Z}_{p}^{*}$ with $\operatorname{gcd}(\tilde{r}, q)=1, M \in_{R}\{0,1\}^{*}, k_{[1, n,\{i\}]} \in_{R} \mathrm{Z}_{q}^{*} \times \cdots \times \mathrm{Z}_{q}^{*}, \alpha=$ $f_{\alpha}\left(a_{[1, n,\{i\}]}\right), \alpha_{1}^{\prime}=f_{\alpha_{1}^{\prime}}\left(a_{[1, n,\{i\}]}\right), \alpha_{2}^{\prime}=f_{\alpha_{2}^{\prime}}\left(a_{[1, n,\{i\}]}\right)$, with $f_{\alpha}$ determined by $G_{o}, f_{\alpha_{1}^{\prime}}, f_{\alpha_{2}^{\prime}}$ determined by $G_{f}, P_{i} \in G_{o}, G_{f}$,

Therefore $\mathbf{D L P}_{q} \equiv_{1-t t}^{p}$ Forge $\left[G_{o}, G_{f}\right]$.

This proof does not hold when: (i) $\alpha_{1}^{\prime}=0$, (ii) $\beta^{\prime}$ satisfies $\operatorname{gcd}\left(g^{\beta^{\prime}}, q\right)=1$. In both cases we cannot show $\mathbf{D L P}_{q} \leq_{1-t t}^{p}$ Forge $\left[G_{o}, G_{f}\right]$.

A typical example of the latter case is when the signer structure from the first signer to $P_{i}$ in $G_{o}$ is preserved in $G_{f}$. For example, it is easy to forge a signature for $\left.G_{f}=\left(\cdots, P_{i}, P_{i+2}, P_{i+1}, \cdots\right)\right)$ from an original graph $G_{o}=$ $\left(\cdots, P_{i}, P_{i+1}, P_{i+2}, \cdots\right)$. 


\subsection{Constructible and Available Groups}

We give a very rough estimate of the number of signers for which the complete set of signer groups becomes constructible.

Let $T_{n}$ be the number of all signer structures for $n$ signers whose directed graph $G$ satisfies conditions (G-1) and (G-2). Then,

$$
T_{n}<2^{n}\left(2^{n}-1\right)\left(2^{n}-2\right) \cdots\left(2^{n}-(n-1)\right)<2^{n^{2}} .
$$

A bound for the number $K_{n}$ of all partial public keys for $G$ is given by,

$$
K_{n} \leq 2\left(2^{n}-1\right)+1=2^{n+1}-1 .
$$

Therefore, the number of all partial public keys for all directed graphs with $n$ signers is bounded by,

$$
T_{n} \cdot K_{n}<2^{n^{2}}\left(2^{n+1}-1\right)=2^{n^{2}+n+1}-2^{n^{2}} .
$$

Using the birthday paradox we can now get an upper bound on the number of signers for which we have constructible groups for any set. We have

$$
T_{n} \cdot K_{n}<2^{n^{2}+n+1}-2^{n^{2}}<<\sqrt{q} .
$$

Thus when $n<\sqrt{\log \sqrt{q}}$ any signer group should have a different public key.

Figure 3 and Figure 4 show directed non-series-parallel graphs whose signer groups are not available. The partial public keys of these structures are the same for any choice of signers' secret keys.

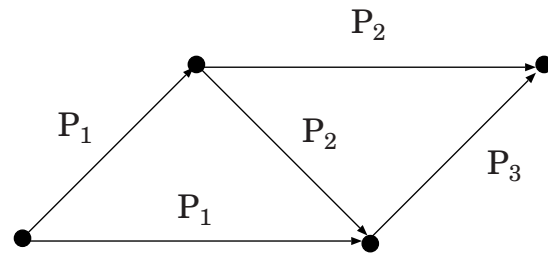

Fig. 3. A non series-parallel graph

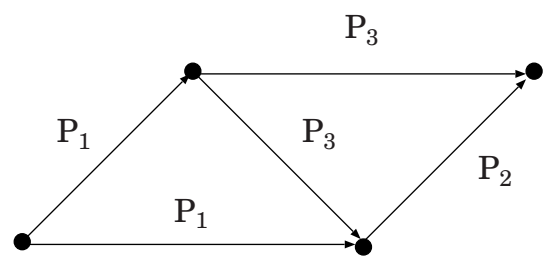

Fig. 4. A non series-parallel graph

Indeed, let $y_{\text {fig. } 3}$ and $y_{\text {fig. } 4}$ be public keys of these graphs respectively. We can easily check that

$$
\begin{aligned}
y_{\text {fig. } 3} & \equiv g^{\left(\left(a_{1}+\left(a_{1}+1\right) a_{2}\right)+1\right) a_{3}+\left(a_{1}+1\right) a_{2}} \\
& =g^{\left(\left(a_{1}+\left(a_{1}+1\right) a_{3}\right)+1\right) a_{2}+\left(a_{1}+1\right) a_{3}} \\
& \equiv y_{\text {fig. } .4} .
\end{aligned}
$$




\section{An Efficient Structured Multisignature Scheme}

We have not fully exploited the structure of the decomposition tree in our earlier approach. We shall now show how this can be done by considering the group structure $G_{f i g 1}=\left[\wedge\left(P_{1},\left(P_{2}, P_{3}, P_{4}\right)\right), \wedge\left(\left(P_{5}, P_{6}\right), P_{7},\left(P_{8}, P_{9}\right)\right)\right]$ in Figure 1. From the decomposition tree in Figure 2, using our earlier approach for serial/parallel executions, we see that may take as group secret key, the key

$$
a=\left[\left[a_{1}+\left(\left(a_{2}+1\right) a_{3}+1\right) a_{4}\right]+1\right] \cdot\left[\left(a_{5}+1\right) a_{6}+a_{7}+\left(a_{8}+1\right) a_{9}\right]
$$

with corresponding group public key $y=g^{a}$. Here a secret key is computed for every sub-tree in Figure 2 precisely once: $\left[a_{1}+\left(\left(a_{2}+1\right) a_{3}+1\right) a_{4}\right]$ is the secret key for the left sub-tree $\left[\wedge\left(P_{1},\left(P_{2}, P_{3}, P_{4}\right)\right]\right.$. This makes use of $\left(\left(a_{2}+1\right) a_{3}+1\right) a_{4}$ which is the secret key for $\left(P_{2}, P_{3}, P_{4}\right)$. Similarly $\left[\left(a_{5}+1\right) a_{6}+a_{7}+\left(a_{8}+1\right) a_{9}\right]$ is the secret key for the right sub-tree $\wedge\left(\left(P_{5}, P_{6}\right), P_{7},\left(P_{8}, P_{9}\right)\right)$ which uses $\left(a_{5}+1\right) a_{6}$ as secret key for $\left(P_{5}, P_{6}\right)$ and $\left(a_{8}+1\right) a_{9}$ as secret key for $\left(P_{8}, P_{9}\right)$.

The resulting scheme is far much more efficient than the earlier scheme for which the group key was,

$$
\begin{aligned}
a^{\prime}= & \left(\left(\left[a_{1}+\left(\left(a_{2}+1\right) a_{3}+1\right) a_{4}\right]+1\right) a_{5}+1\right) a_{6}+ \\
& +\left(\left[a_{1}+\left(\left(a_{2}+1\right) a_{3}+1\right) a_{4}\right]+1\right) a_{7}+ \\
& +\left(\left(\left[a_{1}+\left(\left(a_{2}+1\right) a_{3}+1\right) a_{4}\right]+1\right) a_{8}+1\right) a_{9} .
\end{aligned}
$$

Since the security for the general case can be reduced to that of the serial and parallel structures, we only need to prove that these are secure. This follows from our earlier reductions.

Let us describe the multisignature protocol for the example of the group structure $G_{f i g 1}$ with the decomposition tree shown in Figure 2. The partial public key of $P_{i}$ in $G_{f i g 1}$ is computed from the secret key $a_{i} \in \mathrm{Z}_{q}^{*}$ and other partial keys $y_{j}(j \neq i)$ as follows: $y_{1}=g^{a_{1}}(\bmod p)$ for the first left branch, $y_{2}=g^{a_{2}}(\bmod$ $p), y_{3}=\left(y_{2} g\right)^{a_{3}}(\bmod p), y_{4}=\left(y_{3} g\right)^{a_{4}}(\bmod p)$, for the second left branch, $y_{5}=$ $\left(\left(y_{1} y_{4}\right) g\right)^{a_{5}}(\bmod p), y_{6}=\left(\left(y_{1} y_{4}\right) y_{5} g\right)^{a_{6}}(\bmod p)$ for the first right branch, $y_{7}=$ $\left(\left(y_{1} y_{4}\right) g\right)^{a_{7}}(\bmod p)$, for the second right branch, $y_{8}=\left(\left(y_{1} y_{4}\right) g\right)^{a_{8}}(\bmod p), y_{9}=$ $\left(\left(y_{1} y_{4}\right) y_{8} g\right)^{a_{9}}(\bmod p)$ for the third right branch. Finally the public key $y$ of $G_{f i g 1}$ for the new protocol is computed by $y=y_{6} y_{7} y_{9}(\bmod p)$.

- Computing $r$ : We have

$$
\begin{aligned}
& r_{4}=r_{3}^{a_{4}} g^{k_{4}} \quad(\bmod p), \\
& r_{5}=\left(r_{1} r_{4}\right)^{a_{5}} g^{k_{5}} \quad(\bmod p), \\
& r_{6}=\left(\left(r_{1} r_{4}\right) r_{5}\right)^{a_{6}} g^{k_{6}} \quad(\bmod p) \text {. }
\end{aligned}
$$

Similarly,

$$
\begin{aligned}
& r_{7}=\left(r_{1} r_{4}\right)^{a_{7}} g^{k_{7}} \quad(\bmod p), \\
& r_{8}=\left(r_{1} r_{4}\right)^{a_{8}} g^{k_{8}} \quad(\bmod p), \\
& r_{9}=\left(\left(r_{1} r_{4}\right) r_{8}\right)^{a_{9}} g^{k_{9}} \quad(\bmod p) .
\end{aligned}
$$


Then $r=r_{6} r_{7} r_{9}(\bmod p)$.

- Computing $s$ : We have

$$
\begin{aligned}
& s_{4}=\left(s_{3}+1\right) a_{4}+k_{4} r \cdot \operatorname{hash}(r, M) \quad(\bmod q), \\
& s_{5}=\left(\left(s_{1}+s_{4}\right)+1\right) a_{5}+k_{5} r \cdot \operatorname{hash}(r, M) \quad(\bmod q), \\
& s_{6}=\left(\left(s_{1}+s_{4}\right)+s_{5}+1\right) a_{6}+k_{6} r \cdot \operatorname{hash}(r, M) \quad(\bmod q) .
\end{aligned}
$$

Similarly,

$$
\begin{aligned}
& s_{7}=\left(\left(s_{1}+s_{4}\right)+1\right) a_{7}+k_{7} r \cdot h \operatorname{hash}(r, M) \quad(\bmod q) \text {, } \\
& s_{8}=\left(\left(s_{1}+s_{4}\right)+1\right) a_{8}+k_{8} r \cdot \operatorname{hash}(r, M) \quad(\bmod q) \text {, } \\
& s_{9}=\left(\left(s_{1}+s_{4}\right)+s_{8}+1\right) a_{9}+k_{9} r \cdot \operatorname{hash}(r, M) \quad(\bmod q) \text {. }
\end{aligned}
$$

So $s=s_{6}+s_{7}+s_{9}(\bmod p)$.

The new multisignature of $M$ by $G_{f i g 1}$ is $(s, r)$. It is verified by checking that

$$
g^{s} \stackrel{?}{\equiv} y r^{r \cdot \operatorname{hash}(r, M)}(\bmod p) \text {. }
$$

\section{Conclusions}

A structured multisignature scheme is a scheme for which the group of signers is structured. We have studied such schemes and proposed structured multisignature schemes based on a modified ElGamal signature scheme. For the security proof we have considered attacks for which all the signers except one honest signer $P_{i}$ collude. The attackers give a partial signature for a message $M$ to $P_{i}$ and obtain a valid partial signature for $M$ by $P_{i}$. Under this attack model, the security of the proposed scheme is proven by showing reductions of the discrete logarithm problem to the problems of extracting a secret key of a target signer, changing the signing order in a serial multisignature and changing the signing structure in a mixed structure.

\section{Acknowledgements}

The authors are grateful to Chandana Gamage and Yuliang Zheng for their kind assistance during the printing trouble of this paper.

\section{References}

[BR93] M. Bellare, and P. Rogaway, Random Oracles are Practical: a paradigm for designing efficient protocols, Proc. of 1st ACM Conference on Computer and Communication Security, pp. 62-73, 1993. 468 
[CGH98] R. Canetti, O. Goldreich, and S. Halevi, The Random Oracle Methodology, Revisited, Proc. of the 30th Annual ACM Symposium on Theory of Computing, STOC, pp. 209-218, 1998. 468

[DF91] Y. Desmedt, and Y. Frankel, Shared generation of authenticators and signatures, Lecture Notes in Computer Science 576, Advances in Cryptology -Crypto '91, pp. 457-469, 1991. 477

[DOMU94] H. Doi, E. Okamoto, M. Mambo, and T. Uyematsu, Multisignature Scheme with Specified Order, Proc. of the 1994 Symposium on Cryptography and Information Security, SCIS94-2A, January 27-29, 1994. 467, 477

[DOM98] H. Doi, E. Okamoto, and M. Mambo, Multisignature Schemes for Various Group Structures, The 36-th Annual Allerton Conference on Communication, Control, and Computing, pp. 713-722,1999. 467, 468, 477

[ElG85] T. ElGamal, A public key cryptosystem and a signature scheme based on discrete logarithms, IEEE Trans. on Inform. Theory, Vol. IT-31, No. 4, pp. 469-472, 1985. 467, 472

[FS86] A. Fiat, and A. Shamir, How to Prove Yourself: Practical Solution to Identification and Signature Problems, Lecture Notes in Computer Science 263, Advances in Cryptology -Eurocrypt '86, Spring-Verlag, pp. 186-194 1987. 468

[HMP94] P. Horster, M. Michels, and H. Petersen, Meta-ElGamal Signature Schemes, Proc. of the 2nd ACM Conference on Computer and Communications Security, pp. 96-107, November 1994. 467

[HMP95] P. Horster, M. Michels, and H. Petersen Meta-multisignature schemes based on the discrete logarithm problem, Information Security -the Next Decade, Proc. of IFIP/Sec'95, Chapman \& Hall pp. 128-142 1995. 467

[HZ92] T. Hardjono, and Y. Zheng A practical digital multisignature scheme based on discrete logarithms, Lecture Notes in Computer Science 718, Proc. of Auscrypt'92, Springer-Verlag, pp. 122-132, 1993. 467

[Len90] T. Lengauer, Combinatorial Algorithms for Integrated Circuit Layout, B. G. Teubner Stuttgart, John Wiley \& Sons, 1990. 468

[OO91] K. Ohta, and T. Okamoto, A digital multisignature scheme based on the Fiat-Shamir scheme, Lecture Notes in Computer Science 739, Advances in Cryptology -Asiacrypt'91, Springer-Verlag, pp. 139-148, 1993. 467, 468

[OO99] K. Ohta, and T. Okamoto, Multisignature schemes secure against active insider attacks, IEICE Trans. Fundamentals Vol. E82-A, No. 1, pp. 21-31, 1999. 467

[Oka88] T. Okamoto, A Digital Multisignature Scheme Using Bijective PublicKey Cryptosystems, ACM Trans. on Computer Systems, Vol. 6, No. 8, pp. 432-441, November 1988. 467

[PS96] D. Pointcheval, and J. Stern, Security Proofs for Signature Schemes, Lecture Notes in Computer Science 1070, Advances in Cryptology-Eurocrypt '96, Springer-Verlag, pp. 387-398, 1996. 468

[RSA78] R. L. Rivest, A. Shamir, and L. Adleman, A method for obtaining digital signatures and public-key cryptosystems, Communcation of the ACM, Vol. 21, No. 2, pp. 120-126, 1978. 467

[Schn91] C. P. Schnorr, Efficient Signature Generation by Smart Cards, Journal of Cryptology, Vol. 4, No. 3, pp. 161-174 1991. 467, 474 
[Shim94] A. Shimbo, Multisignature Schemes Based on the ElGamal Scheme, Proc. of The 1994 Symposium on Cryptography and Information Security, SCIS94-2C, January 27-29, 1994. 467

[Woll87] H. Woll, Reductions among number theoretic problems, Information and Computation, Vol. 72, pp. 167-179, 1987. 470

[YL93] S. Yen, and C. Laih, New Digital Signature Scheme Based on Discrete Logarithm, Electronics Letters, Vol. 29, No. 12, pp. 1120-1121, 1993. 472 